\title{
Technical note: Random forests prediction of daily eating time of dairy cows from 3-dimensional accelerometer and radiofrequency identification
}

\author{
Leslie Foldager, ${ }^{1,2 *}$ ๑ Philipp Trénel, ${ }^{3} \odot$ Lene Munksgaard, ${ }^{1} \oplus$ and Peter T. Thomsen ${ }^{1} \odot$ \\ ${ }^{1}$ Department of Animal Science, Aarhus University, DK8830 Tjele, Denmark \\ ${ }^{2}$ Bioinformatics Research Centre, Aarhus University, DK8000 Aarhus C, Denmark \\ ${ }^{3}$ AgroTech, Danish Technological Institute, DK8200 Aarhus N, Denmark
}

\section{ABSTRACT}

Feed intake and time spent eating at the feed bunk are important predictors of dairy cows' productivity and animal welfare, and deviations from normal eating behavior may indicate subclinical or clinical disease. In the current study, we developed a random forests algorithm to predict dairy cows' daily eating time (of a total mixed ration from a common feed bunk) using data from a 3-dimensional accelerometer and a radiofrequency identification (RFID) prototype device (logger) mounted on a neck collar. Models were trained on continuous focal animal observations from a total of 24 video recordings of 18 dairy cows at the Danish Cattle Research Centre (Foulum, Tjele, Denmark). Each session lasted from 21 to $48 \mathrm{~h}$. The models included both the present time signal and observations several seconds back in time (lag window). These time-lagged signals were included with the purpose of capturing changes over time. Because of the high costs of installing an RFID antenna in the feed bunk, we also investigated a model based solely on 3-dimensional accelerometer data. Furthermore, to address the trade-off between prediction accuracy and reduced model complexity and its implications for battery longevity, we investigated the importance of including observations back in time using lag window sizes between 8 and 128 s. Performance was evaluated by internal leave-one-cow-out cross-validation. The results indicated that we obtained accurate predictions of daily eating time. For the most complex model (a lag window size of $128 \mathrm{~s}$ ), the median of the balanced accuracy was 0.95 (interquartile interval: 0.93 to 0.96 ), and the median daily eating time deviation was 7 min $37 \mathrm{~s}$ (interquartile interval: -6 to $15 \mathrm{~min})$. The median of the average daily eating time during sessions was $3 \mathrm{~h} 41 \mathrm{~min}$ with an interquartile

Received September 19, 2019.

Accepted February 4, 2020.

*Corresponding author: leslie@anis.au.dk interval of $2 \mathrm{~h} 56 \mathrm{~min}$ to $4 \mathrm{~h} 16 \mathrm{~min}$. Exclusion of RFID data resulted in a considerable decrease in prediction accuracy, mainly due to a decreased sensitivity of locating the cow at the feed bunk (median balanced accuracy of 0.87 at a lag window size of 128 s). In contrast, prediction accuracy only slightly decreased with decreasing lag window size (median balanced accuracy of 0.94 at a lag window size of $8 \mathrm{~s}$ ). We suggest a lag window size of $64 \mathrm{~s}$ for further development of the prototype logger. The methodology presented in this paper may be relevant for future automatic recordings of eating behavior in commercial dairy herds.

Key words: dairy cow eating time, accelerometer, radiofrequency identification, random forests prediction

\section{Technical Note}

Feed intake of individual cows is important for monitoring health and feed efficiency in dairy cows (De Mol et al., 2016). Although feed intake is difficult to measure at the individual cow level in loose-housing systems, automatic measurements of eating time may be feasible using sensors. Eating time is correlated with feed intake, although this correlation may depend on other factors; for example, feed ration (De Mol et al., 2016). Feeding behavior in dairy cows with access to a common feed bunk occurs in shorter bouts within a meal (Tolkamp et al., 1998). Hence, the accuracy of a prediction algorithm for estimating eating time in dairy cows is expected to depend on the temporal information concerning periods between consecutive visits and data registrations at the feed bunk (Trénel et al., 2009).

Lyngsoe Systems (Aars, Denmark) developed a prototype of an automatic system for measuring dairy cows' eating time consisting of 3-dimensional accelerometers and radiofrequency identification (RFID) sensor devices (CowTrack Logger) mounted on neck collars, and a low frequency field generator at the feed bunk (CowTrack Exciter Box) using a single-wire electric loop placed in the full length of the feed bunk under the feed. This sensor system records activity (accelera- 
tion) and position (yes/no) at the feed bunk (RFID signal) from which we aimed to measure eating time, number of visits at the feed bunk, and daily activity. Data were manually transferred to a computer via a detachable memory stick. In dairy farming, sensor battery longevity optimally spans a cow's lifetime at the farm. Battery longevity depends on factors such as the technology used for data transmission and the amount and complexity of calculations carried out within the sensor. It is, therefore, often crucial to identify a minimal adequate prediction algorithm yielding high prediction accuracy at a modest level of calculation complexity.

The aim of the present study was to test the Lyngsoe Systems prototype sensor in a Danish research herd on a limited number of animals for a shorter duration and to construct a statistical algorithm for estimation of daily eating time (of a TMR from a common feed bunk) from the sensor data. The Danish Holstein cows were kept in a loose housing system, and the feed bunk was equipped with headlocks. We present a random forests model (Breiman, 2001) for estimation of daily eating time and investigate performance and influence of changing parameters of the model by cross-validation. One reason for choosing random forests was the possibility of implementing decision rules directly on later versions of the sensor. This may enable straightforward calculations of daily eating time without the need for time- and battery-consuming transfer of large amounts of raw data. A considerable cost factor of the prototype system is the mounting of the RFID field generator within the feed bunk. Therefore, we also examined the importance of excluding RFID information from the model.

The prototype of the logger was built into a plastic box $(185 \mathrm{~mm} \times 85 \mathrm{~mm} \times 20 \mathrm{~mm})$, which was mounted on the neck collars using a leather bag. The logger consisted of an electronic circuit board containing a 3 -dimensional accelerometer chip, a low frequency detector tag, and a real-time clock running on a button cell battery on the circuit. Data were sampled at a frequency of 12 to $14 \mathrm{~Hz}$, stored in ASCII text files on a 2-GB detachable USB memory stick, and transferred manually to a computer. Two copies of the logger were used for data collection in turn for all cows.

Data from 24 cow/logger combinations were collected and synchronized with video recordings. Each session lasted from 21 to $48 \mathrm{~h}$. Six of 18 cows were measured twice (once with each logger) with sessions using the same cow separated by at least $14 \mathrm{~d}$ and, hence, assumed to be independent. For ease of presentation, we will refer to the cow/logger combinations as cows. On a second basis, video observations were classified through continuous focal animal observation by a trained technician into the following states: ears behind feed bunk, ears above feed bunk, eating (muzzle in contact with the feed or maximum $10 \mathrm{~cm}$ above the feed), other, or view blocked. This classification was then dichotomized into "eating" and "not eating." Logger and video data were time synchronized and averaged to 1 observation per second. The RFID signal was dichotomized: 0 if no signals were detected within $1 \mathrm{~s}$, and 1 otherwise. In total, records of $2,864,478 \mathrm{~s}$ were obtained from the 24 cows.

We developed random forests algorithms for prediction of daily eating time using observations at the present time and several seconds back in time (lag windows). Before data modeling, features of the input data were extracted by calculating time-lagged observations of the accelerometer data with a window size $k$ that varied between 8 and $128 \mathrm{~s}$. Random forests is an ensemble of classification trees with each tree being a set of decision rules (Breiman 2001, 2003). Calculations were carried out using the "randomForest" package (Liaw and Wiener, 2002) in $\mathrm{R}$ version 3.1.0 ( $\mathrm{R}$ Core Team, 2014). The Appendix describes the random forests algorithm in more detail. We examined the effects of the number of trees by increasing from 50 trees to 75 and 100 trees.

We evaluated performance and impact of the lag window size and number of trees by a "leave-one-cowout" cross-validation strategy; that is, preserving in turn data from 1 cow as the test set and using data from the remaining 23 cows for training of the random forests model. Performance was assessed by calculating median and interquartile intervals of sensitivity, specificity, balanced accuracy, and average deviation from the daily eating time determined from video recordings across all "leave-one-cow-out" runs.

In the following, we present random forests models with the formula notation used in $\mathrm{R}$ (Liaw and Wiener, 2002); that is, $Y \sim v_{1}+v_{2}+\ldots+v_{m}$, where $Y$ is the response of interest and $v_{1}, \ldots, v_{m}$ are the $m$ explanatory variables used for building random forests.

Let $\left(x_{t}, y_{t}, z_{t}\right)$ denote the per second-averaged 3-dimensional accelerometer data at time $t$. The corresponding time-lagged observations will be denoted by, for example, $x_{\{t-1\}}, x_{\{t-2\}}, \ldots, x_{\{t-k\}}$, where $t$ is the present time and $k$ is the lag window size; that is, seconds back in time. Let $r_{t}$ denote the corresponding dichotomized RFID signal (0/1, i.e., no/yes being registered at the feed bunk) and $Y_{\text {eating, } t}$ denote the eating status $(0 / 1$, i.e., no/yes) at time $t$ obtained from video recordings. Furthermore, we kept track of time spent in the current RFID state, which was denoted $s_{t}$ (see Supplemental Figure S1; https://doi.org/10.3168/jds.2019-17613).

Note that $s_{t}$ is a stepwise increasing function counting the number of seconds that the RFID has been 0 or 1 , which is reset to 1 every time $r_{t}$ changes from 0 to 1 or from 1 to 0 . We used the following model: 


$$
\begin{aligned}
Y_{\text {eating, } t} & \sim x_{t}+y_{t}+z_{t}+r_{t}+s_{t}+\sum_{j=1}^{k} x_{t-j}+y_{t-j} \\
& +z_{t-j}+r_{t-j}+s_{t-j} .
\end{aligned}
$$

The use of an RFID loop implies extra costs. We therefore also examined model [1] without the RFID measures included (model [2]).

The results indicated that daily eating time in dairy cows was accurately predicted in the current study by combining the prototype sensor system with a random forests modeling strategy, including time-lagged activity variables and an RFID waiting time variable (model [1], Table 1, and Figure 1). A median balanced accuracy of 0.95 with an interquartile interval between 0.93 and 0.96 was found for the most complex model (model [1], lag window size of $128 \mathrm{~s}$ ). For this model, the median daily eating time deviation was $7 \mathrm{~min} 37 \mathrm{~s}$ of underestimation, with an interquartile interval between -6 and 15 min. Deviations in percentage of observed average daily eating time are shown in Supplemental Figure S2 (https://doi.org/10.3168/jds.2019-17613). The median percentage of underestimation for the most complex model was $3.5 \%$, with an interquartile interval between $3.4 \%$ overestimation and $6.5 \%$ underestimation.

Accuracy was found to be highly affected by the exclusion of the RFID-related variables (model [2]) with the median balanced accuracy dropping to 0.87 at a lag window size of $128 \mathrm{~s}$. In contrast, prediction accuracy declined only slightly with diminishing lag window size, showing a median balanced accuracy of 0.94 at lag window size of $8 \mathrm{~s}$ (Table 1). Based on these findings and the visible stop of improvement in terms of shorter whiskers (Figure 1), we suggest a lag window size of 64 s. Number of trees only affected results slightly, and 50 trees was sufficient, except perhaps for the smallest lag window size (Figure 1).

Our findings are in line with published studies on monitoring eating behavior. Pastell et al. (2018) found, in a study based on positioning (Ubisense, Cambridge, UK), high accuracy (97.6\%) for prediction of feeding behavior (sensitivity $=95.3 \%$, specificity $=97.9 \%$ ). Borchers et al. (2016) found a moderately correlated concordance correlation $(\mathrm{CCC}=0.82)$ and Pearson correlation $(\mathrm{r}=0.88)$ between visual observations and sensor estimates of feeding for the CowManager SensOor (Agis, Harmelen, the Netherlands) ear tag accelerometer device. Correspondingly, moderately high correlations were found for the Track A Cow (ENGS, Rosh Pina, Israel) system $(\mathrm{CCC}=0.79, \mathrm{r}=0.93)$, which includes both accelerometer technology and proximity to the feed bunk (Borchers et al., 2016). Results of a pilot study by Wang et al. (2018) indicated that combining data from a leg-mounted accelerometer and location sensors increased the ability to differentiate between eating and standing behavior.

Remarkably, we noted 2 cows with reduced prediction accuracies: cow 6612_2 had an underestimated

Table 1. Cross-validation results: median (25th and 75th quartiles in brackets) of sensitivity, specificity, balanced accuracy, and deviation of predicted average daily eating time ${ }^{1}$

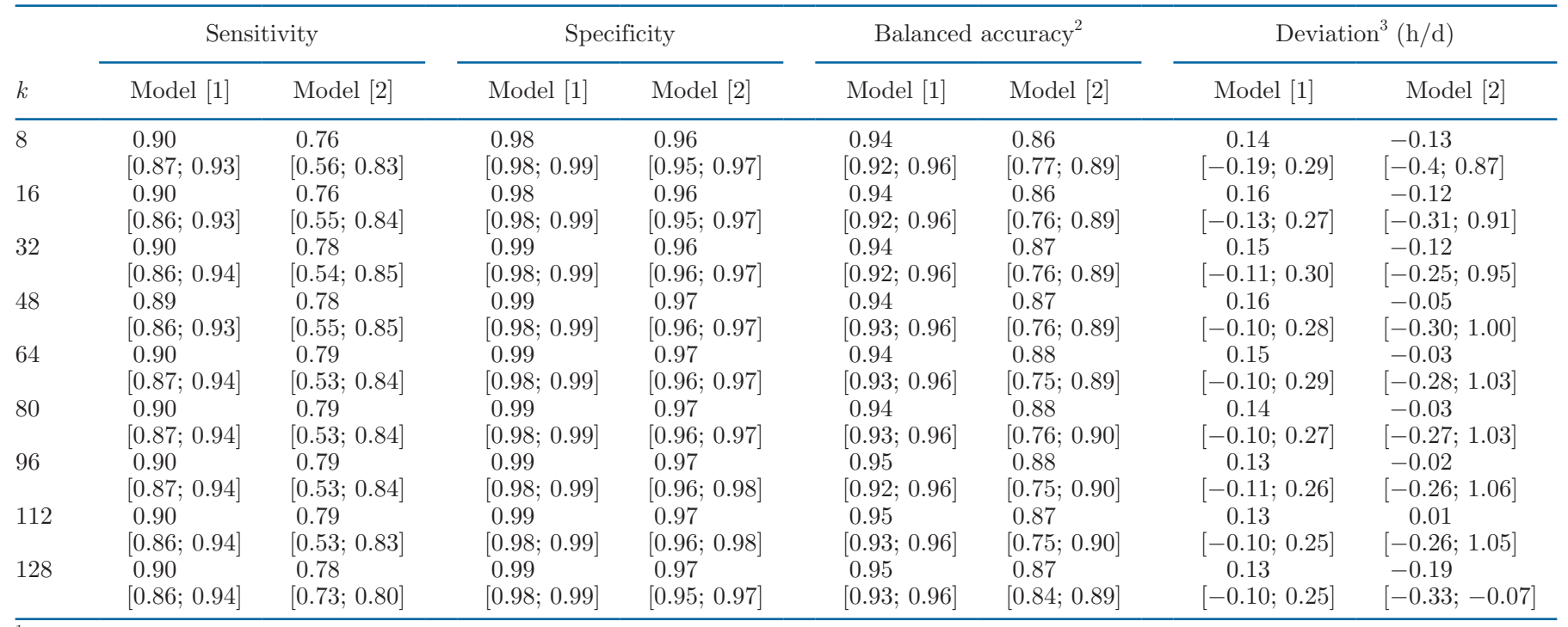

${ }^{1}$ Results are from random forests models with 50 trees and varying lag window sizes $(k)$ in seconds for each of the 2 models including (model [1]) and excluding (model [2]) radiofrequency identification predictors.

${ }^{2}$ Balanced accuracy $=($ sensitivity + specificity $) / 2$.

${ }^{3}$ Deviation in average daily eating time: (true - predicted)/(observation time) [(hours - hours)/(decimal days)]. 

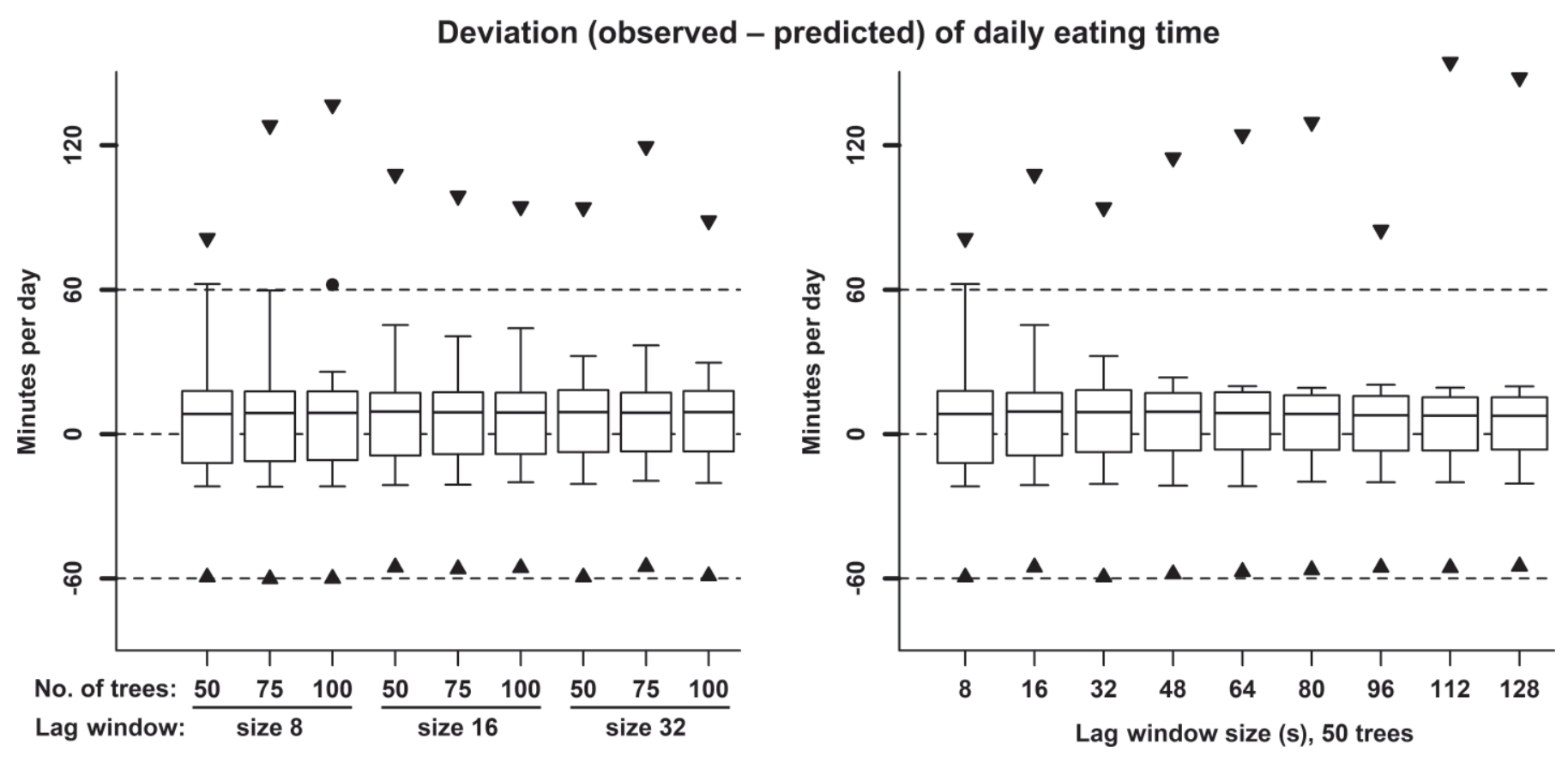

Figure 1. Cross-validation results of comparing the "leave-one-cow-out" cross-validation results from random forests models (including radiofrequency identification measures) for a varied number of trees $(50,75,100)$ and size of lag window (8 to $128 \mathrm{~s})$. Boxplots (R defaults) show median (bar inside), versions of first and third quartile (box), and approximately 1.5 times box length (whiskers). Outlying cows are marked with downward triangles (cow 6758), upward triangles (cow 6612_2), and bullets (cow 2078).

daily eating time and cow 6758 had an overestimated eating time (Figure 1). A closer investigation of the accelerometer coordinates of these 2 cows (Supplemental Figure S3; https://doi.org/10.3168/jds.2019-17613) indicated that the deviating patterns were caused by sudden changes in the coordinate system, probably due to loggers being turned around by the headlocks, as indicated by an examination of the video recordings. This underlines the importance of a robust design of sensor systems to be used in loose housing systems and indicates the need for a gyrometer in the sensor suite to detect or potentially algorithmically correct for sensor orientation; see, for example, Krieger et al. (2018). Considering deviation in percentage of observed average daily eating time, cow 7001_2 also had a relatively large overestimation (Supplemental Figure S2). This cow had the lowest average daily eating time of $1 \mathrm{~h}$ $24 \mathrm{~min}$. The median average daily eating time during sessions was $3 \mathrm{~h} 41 \mathrm{~min}$, with an interquartile interval of $2 \mathrm{~h} 56 \mathrm{~min}$ to $4 \mathrm{~h} 16 \mathrm{~min}$.

The current study demonstrates that daily eating time in dairy cows can be predicted accurately by combining an automatic monitoring sensor system, such as the Lyngsoe Systems prototype used in the current study, with a machine-learning predictive model, such as the random forests modeling strategy applied here. Despite a limited sample size of 24 cows, we demonstrated the importance of careful feature extraction (the inclusion of variables capturing temporal information, including time-lagged activity variables and waiting times between consecutive visits at the feed bunk).

\section{ACKNOWLEDGMENTS}

We are grateful for the opportunity to use the GenomeDK high-performance computing facility at Aarhus University (http://genome.au.dk). We thank the technical staff from the Department of Animal Science, Aarhus University, and the staff at Lyngsoe Systems (Aars, Denmark). CowTrack is a Green Development and Demonstration Programme (GUDP) project conducted in cooperation between Lyngsoe Systems and Department of Animal Science, Aarhus University, with support from The Danish AgriFish Agency, Ministry of Environment and Food. The authors have not stated any conflicts of interest.

\section{REFERENCES}

Borchers, M. R., Y. M. Chang, I. C. Tsai, B. A. Wadsworth, and J. M. Bewley. 2016. A validation of technologies monitoring dairy cow feeding, ruminating, and lying behaviors. J. Dairy Sci. 99:74587466. https://doi.org/10.3168/jds.2015-10843.

Breiman, L. 2001. Random forests. Mach. Learn. 45:5-32. https://doi .org/10.1023/A:1010933404324. 
Breiman, L. 2003. Manual for setting up, using, and understanding random forest v4.0. Accessed Jan. 31, 2020. https://www.stat .berkeley.edu/ breiman/Using_random_forests_v4.0.pdf.

De Mol, R. M., R. M. A. Goselink, J. W. Van Riel, H. M. Knijn, and A. T. M. Knegsel. 2016. The relation between eating time and feed intake of dairy cows. Pages 387-392 in Proc. Precision Dairy Farming 2016, Leeuwarden, the Netherlands. https://doi.org/10 .3920/978-90-8686-829-2.

Krieger, S., G. Sattlecker, F. Kickinger, W. Auer, M. Drillich, and M. Iwersen. 2018. Prediction of calving in dairy cows using a tail-mounted tri-axial accelerometer: A pilot study. Biosyst. Eng. 173:79-84. https://doi.org/10.1016/j.biosystemseng.2017.11.010.

Liaw, A., and M. Wiener. 2002. Classification and regression by randomForest. $\mathrm{R}$ News 2:18-22. https://cran.r-project.org/doc/ Rnews/Rnews_2002-3.pdf.

Pastell, M., L. Frondelius, M. Järvinen, and J. Backman. 2018. Filtering methods to improve the accuracy of indoor positioning data for dairy cows. Biosyst. Eng. 169:22-31. https://doi.org/10.1016/j .biosystemseng.2018.01.008.

R Core Team. 2014. R: A language and environment for statistical computing. R Foundation for Statistical Computing, Vienna, Austria. https://www.R-project.org/.

Tolkamp, B. J., D. J. Allcroft, E. J. Austin, B. L. Nielsen, and I. Kyriazakis. 1998. Satiety splits feeding behaviour into bouts. J. Theor. Biol. 194:235-250. https://doi.org/10.1006/jtbi.1998.0759.

Trénel, P., M. B. Jensen, E. L. Decker, and F. Skjøth. 2009. Technical note: Quantifying and characterizing behaviour in dairy calves using the IceTag automatic recording device. J. Dairy Sci. 92:33973401. https://doi.org/10.3168/jds.2009-2040.

Wang, J., Z. He, G. Zheng, S. Gao, and K. Zhao. 2018. Development and validation of an ensemble classifier for real-time recognition of cow behavior patterns from accelerometer data and location data. PLoS One 13:e0203546. https://doi.org/10.1371/journal .pone. 0203546 .

\section{ORCIDS}

Leslie Foldager (• https://orcid.org/0000-0002-2639-826X Philipp Trénel @ https://orcid.org/0000-0002-4859-1087

Lene Munksgaard @ https://orcid.org/0000-0002-6343-0425

Peter T. Thomsen () https://orcid.org/0000-0002-0896-1610

\section{APPENDIX}

Here, we give a short description of the steps of the random forests algorithm (Breiman, 2001, 2003; Liaw and Wiener, 2002). For a technical presentation and introduction to random forests, we refer to Breiman
(2001). For each of a prespecified number of trees, a bootstrap sample is drawn from the original data by sampling with replacement. These bootstrap samples have the same size $(\mathrm{n}=2,864,478)$ as the original data but contain, on average, approximately two-thirds of the individual records because some records are selected more than once and some are not selected at all (equation $[\mathrm{A} 1])$ :

$$
\begin{aligned}
& P(\text { record } j \text { picked at least once }) \\
& =1-P(\text { record } j \text { never picked }) \\
& =1-\left(\frac{N-1}{N}\right)^{N} \underset{N \rightarrow \infty}{\rightarrow} 1-e^{-1} \cong 0.6321,
\end{aligned}
$$

where $P$ denotes probability and $N$ is sample size. This bootstrap sample is used for training an "unpruned" classification tree. At each node of the tree, several predictors are chosen at random as candidates for splitting the data present at the current node into 2 chunks. Except for the use of 50 trees, we used the default settings of "randomForest" for binary classification, which includes picking the square root of the number of predictors at each node. Initial hyperparameter tuning indicated that this default option regarding number of variables picked per tree $[$ mtry $=\operatorname{sqrt}(\mathrm{n})]$ performed best. The algorithm then chooses the candidate and cut-point (if continuous) that gives the largest reduction of the Gini index; that is, the most homogeneous child nodes in terms of the outcome to be predicted. Each tree is grown as large as possible. The random selection of candidate predictors at each node protects from overfitting (Breiman, 2001) and no pruning is needed. Once the random forests collection of trees has been obtained, a new record can be run through each decision tree, and majority votes are used to predict the class of the new data. 